\title{
Examination of polymorphic glutathione S-transferase (GST) genes, tobacco smoking and prostate cancer risk among Men of African Descent: A case-control study
}

\author{
Nicole A Lavender ${ }^{1,2}$, Marnita L Benford ${ }^{1,2}$, Tiva T VanCleave ${ }^{1,2}$, \\ Guy N Brock $2,3,4,5$, Rick A Kittles ${ }^{6}$, Jason H Moore ${ }^{7}$, David W Hein 1,2,4,5 and \\ La Creis R Kidd*1,2,8
}

\begin{abstract}
Address: ${ }^{1}$ Department of Pharmacology \& Toxicology, University of Louisville (UofL), School of Medicine, 500 South Preston Street, Room 1319 Research Tower, UofL Health Science Center, Louisville, KY 40202, USA, 2James Graham Brown Cancer Center, Cancer Prevention \& Control Program, UofL, 529 South Jackson Street, Louisville, KY 40202, USA, ${ }^{3}$ Department of Bioinformatics \& Biostatistics, UofL School of Public Health and Information Sciences, 485 East Gray Street, Louisville, KY 40202, USA, ${ }^{4}$ Center for Genetics and Molecular Medicine, UofL School of Medicine, Delia B. Baxter Biomedical Research Building, Suite 221, Louisville, Kentucky 40202, USA, ${ }^{5}$ Center for Environmental Genomics and Integrative Biology, UofL, Delia B. Baxter Biomedical Research Building, Suite 221, Louisville, Kentucky 40202, USA, ${ }^{6}$ Department of Medicine, University of Chicago, 5841 S. Maryland Ave, MC Chicago, Illinois 606037, USA, ${ }^{7}$ Dartmouth -Hitchcock Medical Center, Dartmouth Medical School, 706 Rubin Building, HB 7937 One, Medical Center Drive, Dartmouth-Hitchcock Medical Center Lebanon, NH 03756, USA and ${ }^{8}$ Department of Epidemiology and Public Health, UofL School of Public Health and Information Sciences, 485 East Gray Street, Louisville, KY 40202, USA
\end{abstract}

Email: Nicole A Lavender - nalave01@louisville.edu; Marnita L Benford - mlbenf01@louisville.edu;

Tiva T VanCleave - tmtemp01@louisville.edu; Guy N Brock - guy.brock@louisville.edu; Rick A Kittles - rkittles@medicine.bsd.uchicago.edu; Jason H Moore - jason.h.moore@dartmouth.edu; David W Hein - dwhein01@gwise.louisville.edu; La Creis R Kidd* - lrkidd01@gwise.louisville.edu

* Corresponding author

Published: 16 November 2009

BMC Cancer 2009, 9:397 doi:10.1 186/147|-2407-9-397
Received: 13 July 2009

Accepted: 16 November 2009

This article is available from: http://www.biomedcentral.com//47/-2407/9/397

(c) 2009 Lavender et al; licensee BioMed Central Ltd.

This is an Open Access article distributed under the terms of the Creative Commons Attribution License (http://creativecommons.org/licenses/by/2.0), which permits unrestricted use, distribution, and reproduction in any medium, provided the original work is properly cited.

\begin{abstract}
Background: Polymorphisms in glutathione S-transferase (GST) genes may influence response to oxidative stress and modify prostate cancer (PCA) susceptibility. These enzymes generally detoxify endogenous and exogenous agents, but also participate in the activation and inactivation of oxidative metabolites that may contribute to PCA development. Genetic variations within selected GST genes may influence PCA risk following exposure to carcinogen compounds found in cigarette smoke and decreased the ability to detoxify them. Thus, we evaluated the effects of polymorphic GSTs ( $M I, T I$, and $P I)$ alone and combined with cigarette smoking on PCA susceptibility.

Methods: In order to evaluate the effects of GST polymorphisms in relation to PCA risk, we used TaqMan allelic discrimination assays along with a multi-faceted statistical strategy involving conventional and advanced statistical methodologies (e.g., Multifactor Dimensionality Reduction and Interaction Graphs). Genetic profiles collected from 873 men of African-descent (208 cases and 665 controls) were utilized to systematically evaluate the single and joint modifying effects of GSTM I and GSTTI gene deletions, GSTPI I05 Val and cigarette smoking on PCA risk.

Results: We observed a moderately significant association between risk among men possessing at least one variant GSTPI $105 \mathrm{Val}$ allele $(O R=1.56 ; 95 \% \mathrm{Cl}=0.95-2.58 ; p=0.049)$, which was confirmed by MDR permutation testing $(p=0.00 \mathrm{I})$. We did not observe any significant single gene effects among GSTMI (OR $=1.08 ; 95 \% \mathrm{Cl}=0.65-1.82 ; \mathrm{p}=0.7 \mathrm{I})$ and $G S T T I(\mathrm{OR}=1.15 ; 95 \% \mathrm{Cl}=$
\end{abstract}


0.66-2.02; $\mathrm{P}=0.622$ ) on PCA risk among all subjects. Although the GSTMI-GSTPI pairwise combination was selected as the best two factor LR and MDR models $(p=0.0 \mathrm{I})$, assessment of the hierarchical entropy graph suggested that the observed synergistic effect was primarily driven by the GSTPI Val marker. Notably, the GSTM I-GSTPI axis did not provide additional information gain when compared to either loci alone based on a hierarchical entropy algorithm and graph. Smoking status did not significantly modify the relationship between the GST SNPs and PCA.

Conclusion: A moderately significant association was observed between PCA risk and men possessing at least one variant GSTPI $105 \mathrm{Val}$ allele $(p=0.049)$ among men of African descent. We also observed a 2.I-fold increase in PCA risk associated with men possessing the GSTPI (Val/Val) and GSTMI $(* I / * I+* I / * 0)$ alleles. MDR analysis validated these findings; detecting GSTPI I05 Val $(p=0.00 \mathrm{I})$ as the best single factor for predicting PCA risk. Our findings emphasize the importance of utilizing a combination of traditional and advanced statistical tools to identify and validate single gene and multi-locus interactions in relation to cancer susceptibility.

\section{Background}

Even though prostate cancer (PCA) ranks the highest in incidence and second mortality among all cancers affecting American men, its etiology and ethnic disparities are largely unknown[1]. For instance, age and family history are the strongest risk factors for PCA, but African-American (AA) men are more than twice as likely to develop the disease compared to other racial or ethnic groups[1]. AA men are also more likely to be diagnosed with PCA at a younger age, with more aggressive disease and poorer prognosis [1-3]. Despite increases in five year survival rates for AA men over the last few decades; their rates still lag far behind other races[1]. While, the reasons for this disparity are largely speculative; risk, incidence, and mortality rates suggest that genetic factors play an important role in PCA initiation and progression[1]. However, lifestyle habits (e.g., cigarette smoking, diet) have also been implicated to increase risk; indicating that environmental factors may contribute to PCA $[1,2,4]$. Therefore, prostate carcinogenesis and its disparity most likely involve a complex interplay between genetic and environmental factors. More specifically, variations in carcinogen metabolism genes may play a critical role in PCA development due to their activation or detoxification functions.

The glutathione S-transferase (GST) gene superfamily encodes enzymes that catalyze the conjugation of glutathione to electrophilic compounds[5,6]. These enzymes generally detoxify endogenous and exogenous agents, but also participate in the activation and inactivation of oxidative metabolites of carcinogenic compounds associated with prostate cancer[7,8]. Variant GST alleles have been identified within the general population. The most extensively studied variant GSTs include two GST deletion alleles (i.e., GSTM1*0/*0 [GenBank: BC024005.2] and GSTT1*0/*0 [GenBank: BC007065.1]) and the GSTP1 Val allele which is characterized by an adenine to guanine substitution at position $-313\left(\mathrm{~A}^{-313} \mathrm{G}\right)$ in exon 5 [GenBank: BC010915.1; dbSNP: rs1695][9]. The functional consequences of the GSTM1 and GSTT1 $\left({ }^{*} 0 /{ }^{*} 0\right)$ genotypes are obvious in terms of enzyme activity; gene deletion results in loss of conjugation potential. The GSTP1 polymorphism, resulting from an isoleucine to valine substitution within the active site of the enzyme at codon $105\left(\mathrm{I}^{105} \mathrm{~V}\right)$, is linked to altered substrate-specific thermostability and conjugation activity [10-12]. For instance, the GSTP1 $105 \mathrm{Val}$ variant has been associated with lower efficiency for diol epoxides of some polycyclic aromatic hydrocarbons, therefore resulting in decreased detoxification of these compounds compared to the Ile allele[10].

Genetic variations in polymorphic GST genes have been implicated in the etiology of numerous cancers [13-18]. Some studies indicate GST polymorphisms are associated with prostate cancer; however, this association is not accepted across all observational studies [18-25]. Discrepancies may be partially attributed to failure to consider gene combinations or interactions with tobacco smoking. Furthermore, few studies have involved a sufficient number of African-Americans in order to adequately investigate the role of GST polymorphisms and environmental factors in PCA development[12,26,27].

To clarify the role of GST genes in PCA risk within a highrisk sub-group, we evaluated the individual and joint modifying effects of three commonly studied sequence variants in a case-control study of 208 cases and 665 disease-free controls among a population of men of African descent. In an exploratory analysis, we evaluated genegene interactions using an available non-parametric statistical model, namely Multifactor Dimensionality Reduction (MDR). This advanced statistical tool readily overcomes sample size limitations often encountered by parametric statistical methods (e.g., logistic regression analysis). MDR has greater than $80 \%$ statistical power and rigor to evaluate gene-gene interactions even in the presence of small sample sizes (i.e., 200 cases and 200 controls). This computationally sound statistical tool enabled 
evaluations of single and multi-locus genetic and environmental markers as indicators of PCA susceptibility within our population sample.

\section{Methods}

\section{Study population}

Unrelated male residents $(\mathrm{n}=1016)$ of Washington D.C. and Columbia SC, were considered for eligibility in the current PCA case control study. Study participants ( $\mathrm{n}=$ 132) were not considered in the current study if they met one or more of the following exclusion criteria: (1) they were diagnosed with benign prostatic hyperplasia $(\mathrm{n}=$ $64) ;(2)$ had an abnormal prostate specific antigen (PSA) and digital rectal examination (DRE) $(\mathrm{n}=11)$; and (3) had European ancestry based on a Global Ancestry score of $<25 \%(n=70)$ [28]. Eligible men of African descent (i.e., self-identified African-Americans, East Africans, West Africans, and Afro-Caribbeans), including 208 patients (ages 41-91) and 665 healthy volunteers (ages 26-89), were recruited from the Howard University Hospital (HUH) Division of Urology PCA patient population, the
HUH PCA screening program, and the South Carolina PCA screening program (Table 1). The PCA patients and screening participants, recruited between 2001 and 2005, had a PSA and DRE. Subjects who had an abnormal PSA $(>4.0 \mathrm{ng} / \mathrm{ml})$ and/or irregular DRE underwent multiple core needle biopsies. Incident PCA cases in the current study were identified by an HUH urologist based on abnormal PSA (>2.5 ng/ml) and/or DRE as well as histological findings following a radical prostatectomy. Inclusion criteria of controls were men with PSA levels $\leq 2.5 \mathrm{ng} /$ $\mathrm{ml}$ and/or normal DREs/biopsies. Tumor grade, ranging from $4-10$, was collected for $62.0 \%$ of the cases $(n=129)$. Subjects $(\mathrm{n}=216)$ were classified as current $(\mathrm{n}=38)$, former $(\mathrm{n}=71)$ and never $(\mathrm{n}=107)$ smokers. All study participants had available DNA extracted from whole blood and provided written informed consent for participation in genetic analysis studies under a protocol approved by the Howard University Institutional Review Board as well as from the HUH Division of Urology.

Table I: Patient and Tumor Characteristics

\begin{tabular}{|c|c|c|c|}
\hline Characteristics & Cases & Controls & p-value ${ }^{a}$ \\
\hline Number of Participants, $n$ & 208 & 665 & --- \\
\hline \multicolumn{4}{|l|}{ Age (yrs) } \\
\hline Median (range) & $65(4|-9|)$ & $52(26-89)$ & 0.0001 \\
\hline \multicolumn{4}{|l|}{ PSA in ng/ml n (\%) } \\
\hline$<4$ & $43(22.0)$ & $609(94.4)$ & $<0.0001$ \\
\hline$\geq 4$ & $152(78.0)$ & $36(5.6)$ & \\
\hline \multicolumn{4}{|l|}{ Gleason Score n (\%) } \\
\hline 4 & $17(13.2)$ & & \\
\hline 5 & $15(11.6)$ & & \\
\hline 6 & $33(25.6)$ & & \\
\hline 7 & $40(31.0)$ & & \\
\hline 8 & $6(4.6)$ & & \\
\hline 9 & $14(10.9)$ & & \\
\hline 10 & $4(3.10)$ & & \\
\hline \multicolumn{4}{|l|}{ Smokers n (\%) } \\
\hline Never & $62(45.6)$ & $45(20.8)$ & 0.162 \\
\hline Former & $51(37.5)$ & $20(25.0)$ & \\
\hline Current & $23(16.9)$ & $15(18.8)$ & \\
\hline \multicolumn{4}{|l|}{ Eversmokers n (\%) } \\
\hline No & $62(45.6)$ & $45(56.3)$ & 0.130 \\
\hline Yes & $74(54.4)$ & $35(43.8)$ & \\
\hline $\begin{array}{l}\text { Global West African Ancestry } \\
\text { Median (SD) }\end{array}$ & $0.791(0.253-0.947)$ & $0.718(0.255-0.946)$ & 0.020 \\
\hline
\end{tabular}

Abbreviations: PSA, prostate specific antigen

aDifferences in frequencies were tested by a Chi-square test of heterogeneity (i.e., PSA ng/ml); Differences in age and Global West African Ancestry between cases and controls were tested using the Wilcoxon sum Rank test. 


\section{TaqMan allelic discrimination of GSTMI, GSTPI and GSTTI sequence variants}

Polymorphisms in three glutathione S-transferases genes were ascertained using TaqMan Polymerase Chain Reaction (PCR) allelic discrimination assays. The following alleles were detected: (1) GSTM1 $\left({ }^{*} 1 /{ }^{*} 1+{ }^{*} 1 /{ }^{*} 0\right)$; (2) GSTT1 (*1/*0 $\left.{ }^{*} 1 /{ }^{*} 0\right)$; and (3) GSTP1 (Ile105Val). The albumin reference gene served as a positive control for GST (M1, T1) deletions. The discrimination assay contained approximately $40 \mathrm{ng}$ of germ-line DNA, $1 \times$ Universal Master Mix (Applied Biosystems), $900 \mathrm{nM}$ of each primer (forward and reverse), and $400 \mathrm{nM}$ of each probe (FAM and VIC) to comprise a $10 \mu \mathrm{l}$ reaction. The primers and probes used to detect a deletion or single nucleotide polymorphisms (SNP) detected in GSTM1, GSTP1 and GSTT1 alleles were obtained using the NCI SNP500 database, published reports and Primer Express 3.0 software (Applied Biosystems, Foster City, CA) [29-31]. The PCR amplification conditions consisted of the following: an initial 2 step hold $\left(50^{\circ} \mathrm{C}\right.$ for $2 \mathrm{~min}$, followed by $95^{\circ} \mathrm{C}$ for $10 \mathrm{~min})$ and 40 cycles of a two-step PCR $\left(95^{\circ} \mathrm{C}\right.$ for $15 \mathrm{~s}$, $60^{\circ} \mathrm{C}$ for $1 \mathrm{~min}$ )[30]. The fluorescent intensity emitted from the fluorogenic probes were measured using the ABI $7900 \mathrm{HT}$ sequence detection system and assigned genotypes using SDS 2.2.1 software (Applied Biosystems, Foster City, CA). To minimize misclassification bias, laboratory technicians were blinded to the case status of study participants. Based on 24 non-DNA template controls per batch analysis, percent cross-contamination during sample handling was negligent $(<0 \%)$. Duplicate genotyping performed on 72 randomly selected samples for quality control purposes resulted in concordance rates of $96-97 \%$ for GST (M1, T1) and $100 \%$ for GSTP1. Call rates were $>94 \%$ for the GST deletion $(M 1, T 1)$ and GSTP1 allelic discrimination assays. In addition, we tested whether the aforementioned GST polymorphisms were in Hardy-Weinberg equilibrium among controls using a significance level of $\mathrm{p}<0.05$.

\section{Ancestry markers}

One hundred previously validated autosomal ancestry markers were included to account for potential population stratification among our admixed population of selfreported African-Americans, West African, East African, Afro-Caribbean, as previously described[32]. Study participants were grouped from lowest to highest genetic West African Ancestry (WAA), with scores ranging from 0$100 \%$. These 100 markers were assembled using DNA from self-identified African-Americans (Coriell Institute for Medical Research, $\mathrm{n}=96$ ), Yoruban West Africans (HapMap, $\mathrm{n}=60$ ), West Africans (Bantu and Nilo Saharan speakers, $n=72$ ), Europeans (New York City, $n=24$ ), and CEPH Europeans (HapMap Panel, $n=60$ ), as previously reported[32]. Individuals $(n=873)$ with a high degree of
WAA greater than or equal to $25 \%$ were considered in the final analysis.

\section{Evaluation of individual GST loci and PCA risk using $L R$ analysis}

To assess whether inheritance of at least one GST (M1, T1) deletion or GSTP1 Val allele was associated with elevated risk of developing PCA, we tested for significant differences in the distribution of variant genotypes between 208 cases and 665 controls using the chi-square test of homogeneity. Associations between PCA risk and candidate polymorphic genes, expressed as odds ratios (ORs) and corresponding 95\% confidence intervals (CIs), were estimated using unconditional multivariate logistic regression (LR) models adjusted for potential confounders [age (yrs), PSA (ng/ml), and WAA (continuous)]. All reported risk estimates and 95\% CIs for the selected polymorphic GST genes used the following as reference genotypes: $\operatorname{GSTM} 1\left({ }^{*} 1 /{ }^{*} 1+{ }^{*} 1 /{ }^{*} 0\right), \operatorname{GSTT} 1\left({ }^{*} 1 /{ }^{*} 1+{ }^{*} 1 /{ }^{*} 0\right)$, GSTP1 (Ile/Ile). Test for trend included genotypes as ordinal variables. Statistical significance was assessed using a p-value $<0.05$. All chi-square test and LR analyses were conducted using SAS 9.1.3 (SAS Institute, Cary, NC).

\section{Gene combination effects}

MDR was used to evaluate gene-gene interactions associated with PCA risk. This tool aids in the identification of high-risk markers using a cross validation strategy to estimate the classification and prediction accuracy of individual and multifactor models [33-35]. MDR is a data mining platform that readily overcomes sample size limitations often encountered by parametric statistical methods (e.g., LR analysis) by collapsing high-dimensional genetic data into a single dimension [33-35]. Also, MDR is a "modelfree" (it does not assume a specified genetic model) and "non-parametric" method (it does not estimate parameters) that is effective with relatively small sample sizes [3335]. With MDR, we pooled multi-locus factors into highrisk and low-risk groups, thereby reducing the otherwise high-dimensional data to a single variable and permitting an investigation of individual GST genes, gene combination effects, and GST-tobacco smoking interactions. This one-dimensional multi-locus variable was evaluated for its ability to classify and predict PCA susceptibility through cross-validation and permutation testing. MDR was utilized to generate a single model that maximizes the number of individuals with the proper risk assignment. Single best factor models were selected among each of the one- or two- factor combinations based on those that minimize the prediction error as well as maximize cross validation consistency (CVC) and average testing accuracy (ATA). To evaluate the number of times the same individual loci or set of genes was identified in each possible 9/ $10^{\text {ths }}$ of the data, the average CVC (based on a scale from $0-100 \%$ ) from the observed data was compared to the dis- 
tribution of average consistencies under the null hypothesis of no association. Statistically significant single and multi-locus models may be adjusted for potential confounders by placing the MDR output variables into a LR model. Furthermore, validation of models as effective predictors of prostate cancer susceptibility was derived empirically from 1,000 permutations. This approach accounted for multiple testing issues as long as the entire model fitting procedure was repeated for each randomized dataset to provide an opportunity to identify false-positives. We considered MDR permutation results to be statistically significant at the 0.05 level. LR analysis was used to perform two-way interaction models for risk estimates terms identified by MDR.

\section{Interaction Entropy Graphs}

Interaction entropy was used as a third strategy to verify, visualize, and interpret combination effects identified by LR and MDR [36,37]. Interaction entropy uses information gain (IG) to gauge whether interactions between two or more variables provide more information about a class variable relative to each variable considered independently [36] and has been applied to several recent epidemiological studies $[34,36,38,39]$. The colors range from red representing a high degree of synergy (positive information gain), orange a lesser degree, and gold representing independence and a midway point between synergy and redundancy. Blue represents the highest level of redundancy (negative information gain), followed by green.

\section{Results}

The patient and tumor characteristics in the current study are summarized in Table 1 . Cases were significantly older than controls and had higher PSA levels. Although there was a small portion of controls (5.6\%) who had PSA levels that exceeded $4.0 \mathrm{ng} / \mathrm{ml}$, these individuals did not have an abnormal DRE or irregular biopsy. There was a significant difference in median West African genetic ancestry estimates comparing cases and controls ( $\mathrm{p}=$ $0.020)$. Cigarette smoking data was available for approximately $25 \%$ of our study subjects ( $\mathrm{n}=216,136$ cases and 80 controls). These subjects were classified by cigarette smoking: current $(\mathrm{n}=38)$, former $(\mathrm{n}=71)$ and never $(\mathrm{n}=$ 107). Cigarette smoking did not differ significantly ( $\mathrm{p}>$ 0.05 ) between cases and controls.

Within our study set, inheritance of two GST (M1, T1) deletions or at least one GSTP1 105 Val allele was fairly common among controls with frequencies ranging from 17.5-47.9\%, as detailed in Table 2. The genotype frequencies among controls were comparable to those observed in other African descendent sub-groups[26,40,41]. The genotype frequencies among controls did not deviate from expected distributions based on the Hardy-Weinberg equilibrium $(\mathrm{p} \geq 0.138)$.

We evaluated the independent effects of genetic variations in highly variant GST genes in relation to PCA susceptibility using unconditional LR models, as detailed in Table 2. Inheritance of at least one GSTP1 105 Val allele (linked with decreased capacity to conjugate electrophilic compounds) was associated with a 1.6 -fold increase in prostate cancer risk $(\mathrm{OR}=1.56 ; 95 \% \mathrm{CI}=0.95-2.58 ; \mathrm{p}=$ 0.049). The GSTP1 Val loci was also identified as the best single factor predictor of PCA based on a cross-validation consistency of $100 \%$, an average testing accuracy of $53 \%$

Table 2: Association between prostate cancer risk and selected GST gene variations

\begin{tabular}{|c|c|c|c|c|c|c|c|}
\hline Gene & SNP & $\begin{array}{l}\text { Case } \\
\text { n (\%) }\end{array}$ & $\begin{array}{l}\text { Control } \\
\text { n (\%) }\end{array}$ & $\begin{array}{l}\text { Risk Estimate } \dagger \\
\text { OR }(95 \% \mathrm{Cl})\end{array}$ & $\begin{array}{l}\text { Risk Estimatett } \\
\text { Adj OR }(95 \% \mathrm{Cl})\end{array}$ & p-valuettt & p-value for Trend \\
\hline GSTPI & AA (lle/lle) & $55(29.0)$ & $186(32.5)$ & I.00 (Reference) & I.00 (Reference) & & \\
\hline$A^{3 / 3} G$ & AG (lle/Val) & 85 (44.7) & $274(47.9)$ & $1.12(0.77-1.63)$ & $1.09(0.67-1.81)$ & & \\
\hline \multirow[t]{3}{*}{$11 e^{105} \mathrm{Val}$} & $\mathrm{GG}(\mathrm{Val} / \mathrm{Val})$ & $50(26.3)$ & $112(19.6)$ & $1.62(1.04-2.5 \mathrm{I})$ & $1.66(0.94-2.96)$ & $0.14 \mid$ & 0.080 \\
\hline & $A G+G G$ & $140(73.7)$ & $460(80.4)$ & $1.47(1.00-2.15)$ & $1.56(0.95-2.58)$ & 0.049 & \\
\hline & missing & $6(3.2)$ & $35(6.1)$ & & & & \\
\hline GSTMI & $* \mid / * 1+* 1 / * 0$ & |4| (75.0) & $44 \mathrm{I}(76.3)$ & I.00 (Reference) & I.00 (Reference) & & \\
\hline \multirow[t]{2}{*}{ Deletion } & $* 0 / * 0$ & $47(25.0)$ & $137(23.7)$ & $1.07(0.73-15.7)$ & $1.08(0.65-1.82)$ & 0.718 & \\
\hline & missing & $8(4.3)$ & $29(5.0)$ & & & & \\
\hline GSTTI & $*|/ * 1+*| / * 0$ & I $53(8 \mid .0)$ & $482(82.5)$ & I.00 (Reference) & I.00 (Reference) & & \\
\hline \multirow{2}{*}{ Deletion } & $* 0 / * 0$ & $36(19.0)$ & $102(17.5)$ & I.II (0.73-1.69) & $1.15(0.66-2.02)$ & 0.622 & \\
\hline & missing & $7(3.7)$ & $23(3.9)$ & & & & \\
\hline
\end{tabular}

\footnotetext{
†Associations were determined using multivariate logistic regression models to estimate the risk of developing PCA using GSTPI I05 Ile/lle, GSTM I $* \mid / * 1+* 1 / * 0$ and GSTTI * $\mid / * 1+* 1 / * 0$ as the reference genotypes.

††Risk estimates adjusted for age (continuous variable); prostate specific antigen (continuous variable); and West African Ancestry, WAA or admixture (continuous variable).

tttDifferences in the frequency of high-risk and low-risk genotypes between cases and controls were determined using the chi-square test of association with a significance level of 0.05 .
} 
and a 1000-fold permutation testing p-value of 0.001 (Table 3). Upon evaluation of gene-gene combination effects, LR modeling revealed a 2.1-fold increase in PCA risk associated with men possessing the GSTP1 (Val/Val) and GSTM1 $\left({ }^{*} 1 /{ }^{*} 1+{ }^{*} 1 /{ }^{*} 0\right)$ alleles $(\mathrm{OR}=2.11 ; 95 \% \mathrm{CI}=$ 1.07-4.16; $\mathrm{p}$-value for interaction $=0.062)$. Initially, it appeared as though this interaction was a significant PCA predictor following MDR analysis with a statistically significant 1000 -fold permutation testing p-value of 0.01 . However, this gene combination effect was primarily driven by the GSTP1 loci attributed to the lack of additional information gain comparing the GSTM1-GSTP1 axis (IG $=0.04 \%$ ) to either GSTP1 or GSTM1 loci alone (IG $=0.58 \%$ and $0.03 \%$, respectively) (Figure 1 ). Lastly, no significant risk effects were detected among the GST deletion polymorphisms ( $\mathrm{p}=0.622)$ or the GSTP1-GSTP1 and GSTM1-GSTT1 combinations ( $\mathrm{p}=0.557$ and 0.814 , respectively). For our exploratory analysis of gene-environment interactions associated with risk, LR modeling indicated that smoking status did not modify the relationship between the GST SNPs and PCA risk (p for interaction $\geq 0.135$ ), as shown in Table 4 .

\section{Discussion}

Previous studies suggest that oxidative stress and reactive species are becoming increasingly important in prostate carcinogenesis $[4,11,12,42-45]$. If these electrophilic compounds are not removed or reduced to less active forms, they may result in damage to biomolecules (e.g., DNA, proteins, etc.) and ultimately lead to cellular dysfunction or transformation. Consequently, the role of antioxidant enzymes has been commonly investigated in relation to PCA development because of their function in the detoxification of potentially damaging carcinogens and/or reactive oxygen species (ROS)[4,46-48]. The GSTs in particular have been extensively studied since they are able to conjugate a wide range of oxidative substrates $[6,11,43,49,50]$. Variations within these genes can cause a loss or reduction in enzymatic activity and have been associated with increased risk of prostate as well as several other cancers (e.g., colon, breast, and lung) $[6,12,51-53]$.

In the case of PCA, reports have observed that PCA cases possess lower antioxidant enzyme levels in prostate tissues compared to both healthy controls and men with benign prostatic hyperplasia (BPH)[48]. In addition, PCA tissues appear to contain higher amounts of ROS and oxidative DNA damage [46-48]. Some investigators speculate these effects may be attributed to GST genetic polymorphisms (i.e., GSTM $1{ }^{*} 0 /{ }^{*} 0, \operatorname{GSTT} 1{ }^{*} 0 /{ }^{*} 0$, and GSTP1 Val) linked with compromised oxidative repair capacity $[5,12,26,41,43,54]$. The goal of this study was to evaluate GSTM1 and GSTT1 gene deletions, variant GSTP1 105 Val, and cigarette smoking as predictors of PCA risk among 873 men of African descent (208 cases and 665 controls). We hypothesized that individuals who possess one or more genotypes associated with reduced detoxifi-

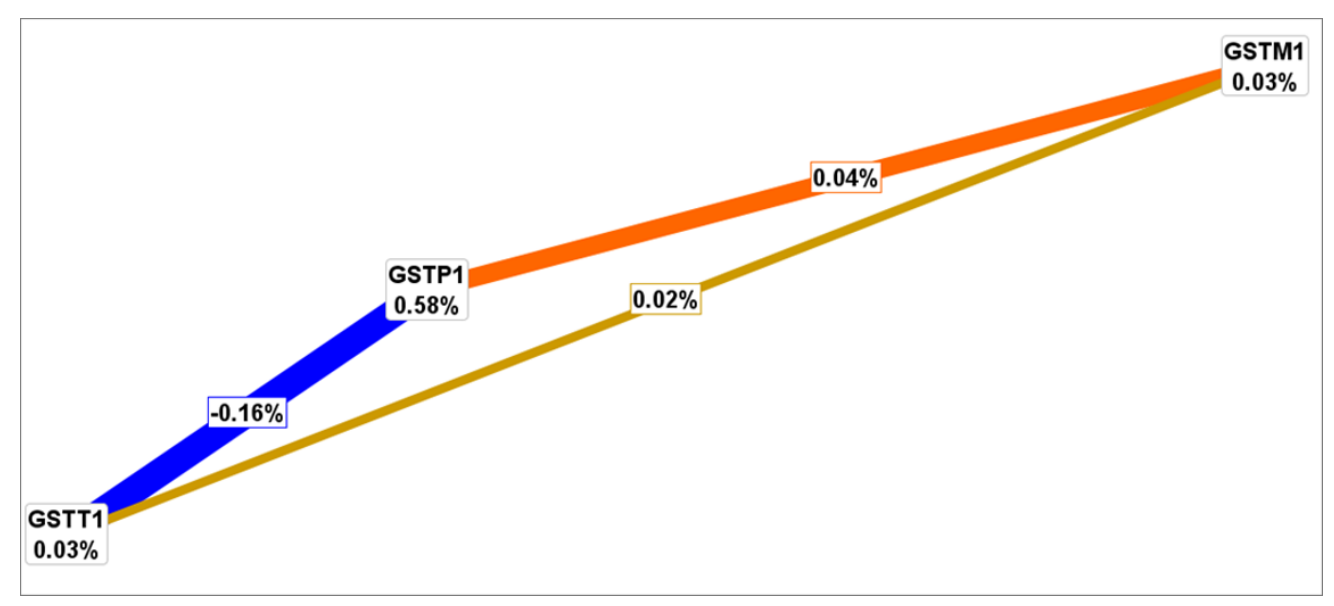

\section{Figure I}

Interaction entropy model for GST gene variations and prostate cancer risk. This graphical model, describes the percent entropy that is explained by each GST SNP or a combination of two loci within our study population. Positive percent entropy indicates information gain or synergy. However, negative percent indicates redundancy or lack of information gain. Schematic coloration used in the visualization tools represents a continuum from synergy (i.e. non-additive) to redundancy. The colors range from red representing a high degree of synergy (positive information gain), orange a lesser degree, and gold representing independence and a midway point between synergy and redundancy. On the other hand, green represents redundancy. Note that the gene combination effect is primarily driven by the GSTPI loci attributed to the lack of additional information gain comparing the GSTMI-GSTPI axis (IG $=0.04 \%$ ) to either GSTPI or GSTMI loci alone $(I G=0.58 \%$ and $0.03 \%$, respectively) 
Table 3: Multifactor Dimensionality Reduction Models for GST gene variations and prostate cancer risk

\begin{tabular}{cccc}
\hline Best Model & Cross Validation Consistency (CVC) & Average Testing Accuracy (ATA) & Permutation Testing p-value \\
\hline $\begin{array}{ccc}\text { One Factor } \\
\text { GSTPI }\end{array}$ & $10 / 10$ & 0.530 & 0.001 \\
\hline $\begin{array}{c}\text { Two Factor } \\
\text { GSTPI } \\
\text { GSTMI }\end{array}$ & $8 / 10$ & 0.535 & 0.01 \\
\hline
\end{tabular}

cation would have a higher risk of developing PCA. In an exploratory analysis, we investigated the effects of cigarette smoking combined with these polymorphisms in relation to PCA risk. This increased risk may be partially attributed to exposure to tobacco carcinogens and altered capacity to metabolically detoxify hazardous compounds. Logistic regression analysis revealed a moderately significant association between PCA susceptibility and GSTP1 $105 \mathrm{Val}$. These findings were confirmed using a robust data-mining tool specifically designed to evaluate main effects and higher order interactions. Although we did not detect any significant single gene effects among GSTM1 and $T 1$ deletions, we did observe a moderately synergistic interaction between GSTP1-GSTM1 and PCA susceptibility. We found a 2.1-fold increase in PCA risk associated with men possessing 2-3 high risk alleles with the GSTP1 GG (Val/Val) \&GSTM $1 * 1 /{ }^{*} 1+{ }^{*} 1 /{ }^{*} 0$ genotype according to logistic regression analysis.

Numerous previous studies have investigated the role of these GST polymorphisms in relation to PCA susceptibility. For example, Agalliu et al. 2006 reported a moderate increase in PCA risk associated with the GSTM1 gene deletion for Caucasian men $(\mathrm{OR}=1.54 ; 95 \% \mathrm{CI}=1.19-2.01)$; however, GSTT1 deletion and GSTP1 105 Val polymorphisms were not significantly associated with prostate cancer[5]. The null findings for GSTT1 and GSTP1 were corroborated in a recent meta-analysis of pooled data from $\geq 3,837$ cases and $\geq 4,552$ controls[12]. This same report, however, observed a 1.3 fold increase in PCA risk among all study participants was associated with the GSTM1*0 loci using pooled data from 4,564 cases and 5,464 controls $(\mathrm{OR}=1.33 ; 95 \% \mathrm{CI}=1.15-1.55)[12]$. Stratification based on self-identified race indicated that risk estimates were comparable between Caucasians and Asians; however, the GSTM1 and GSTT1 deletion polymorphisms did not modify risk among Africans or African-Americans[12]. Interestingly, only two published studies investigated the role of the GSTP1 105 Val SNP in relation to PCA among men of African descent $[5,12,54]$. The null findings in these two reports may be partially attributed to the failure to utilize rigorous statistical tools that permit the evaluation of main effects even in the presence of small sample sizes (i.e., at least 200 cases and 200 controls) such as MDR. The MDR selection of the GSTP1 105 Val loci as a significant PCA predictor in the current study is promising and will undergo subsequent validation within future studies among men of African descent.

We have considered the strengths and limitations of the current study. Although MDR doesn't allow for adjustments of covariates, it does control for multiple compari-

Table 4: Combined effects of GST polymorphisms and smoking on prostate cancer risk

\begin{tabular}{|c|c|c|c|c|}
\hline Gene & Polymorphism & $\begin{array}{l}\text { Adjusted OR }(95 \% \mathrm{Cl}) \text { for Non- } \\
\text { smokers } ¥ \ddagger\end{array}$ & $\begin{array}{l}\text { Adjusted OR }(95 \% \mathrm{Cl}) \text { for Ever- } \\
\text { smokersttt }\end{array}$ & $\mathrm{p}$-value for Interaction \\
\hline GSTPI & AA (lle/lle) \& AG (lle/Val) & I.00 (Reference) & $2.88(0.49-16.82)$ & \\
\hline$A^{3 / 3} G$ & GG $(\mathrm{Val} / \mathrm{Val})$ & $1.91(0.47-7.82)$ & $1.42(0.33-6.10)$ & 0.928 \\
\hline \multicolumn{5}{|l|}{$11 e^{105} \mathrm{Val}$} \\
\hline GSTMI & $*|/ * 1+*| / * 0$ & 1.00 (Reference) & I.7I (0.59-4.97) & \\
\hline Deletion & $* 0 / * 0$ & $1.61(0.36-7.28)$ & $0.47(0.11-2.09)$ & 0.135 \\
\hline GSTTI & $* \mid / * 1+* 1 / * 0$ & 1.00 (Reference) & $1.03(0.37-2.89)$ & \\
\hline Deletion & $*_{0} / * 0$ & $1.02(0.21-4.94)$ & $2.12(0.47-9.57)$ & 0.410 \\
\hline
\end{tabular}

†Associations were determined using multivariate logistic regression models to estimate the risk of developing PCA using GSTPI I05 Ile/lle, GSTM I $* 1 / * 1+* 1 / * 0$ and GSTTI $* 1 / * 1+* 1 / * 0$ as the Reference genotypes.

t†Risk estimates adjusted for age (continuous variable); prostate specific antigen (continuous variable); and West African Ancestry, WAA or admixture (continuous variable).

$\ddagger$ Non-smokers are subjects that have never smoked; Ever-smokers are subjects that are current or former smokers 
sons and spurious risk estimates by using a cross validation and permutation testing scheme as a built-in feature. Misclassification by case status is also a potential limitation for this study. There is a slight possibility that some of the controls with PSA less than $4 \mathrm{ng} / \mathrm{ml}$ may actually have prostate cancer that remains undetected. Controls who had an abnormal PSA $(>4.0 \mathrm{ng} / \mathrm{ml})$ and/or irregular DRE underwent multiple core needle biopsies. Patients with an abnormal biopsy were reclassified as cases. Participants with a PSA (i.e., $\leq 4.0 \mathrm{ng} / \mathrm{ml}$ ) but an abnormal DRE were excluded abnormal DRE were excluded from participating in the current study. These individuals were excluded because we could not predict with any level of certainty whether these individuals would develop prostate cancer. We also excluded individuals who were: (1) diagnosed with BPH following a biopsy; or (2) had an abnormal PSA and/or an irregular DRE. Since one cannot predict with any level of certainty whether individuals diagnosed with $\mathrm{BPH}$ would develop prostate cancer, these individuals were excluded from the current study. As implied within Lobe et al., 2006, even after close inspection of prostate cancer tissue, it is possible to miss a microscopic nodule that can later develop into cancer[55]. If controls in our study population were still misclassified after undergoing a PSA test, DRE, and/or multiple core needle biopsies, then we may expect our calculated risk estimates to underestimate the relationship between the selected GST polymorphisms and prostate cancer susceptibility. But this issue plaques all cancer epidemiology studies. Unfortunately, it is impractical to subject all patients to a radical prostatectomy to permit an extensive evaluation and more accurate classification of case status.

Another challenge for genetic epidemiology studies involving study participants of African descent is their unique population history of gene flow from divergent populations [56,57]. The current study adjusted single loci models for genetic heterogeneity (i.e., population admixture). This also helps to circumvent misclassification of study participants related to self-identified race/ethnicity (SIRE). Our findings suggest that inclusion of WAA did not significantly change the risk estimates relative to unadjusted models; if anything it makes them more precise. Also, this study may be limited due to lack of consideration of the genetic heterogeneity of selected targets. This could be the reason we did not observe any statistical significant effects among GST polymorphisms and smoking interactions in relation to PCA risk $(p=0.105)$.

\section{Conclusion}

In summary, our findings indicate a moderately significant association between risk and among men possessing at least one variant GSTP1 105 Val allele ( $\mathrm{p}=0.049)$ among men of African descent. MDR analysis validated the logistic regression findings and identified the GSTP1105 Val allele ( $\mathrm{p}=0.001)$ as the best single factor model for predicting PCA risk. The ability of MDR to evaluate main effects and remain effective with relatively small sample sizes of at least 200 cases and 200 controls strengthens the power of this study. Statistically significant single and multi-locus models maybe adjusted for potential confounders by placing the MDR output variables into a LR model. Furthermore, this study is robust since it utilizes multiple statistical analysis tools to examine the single gene as well as gene-gene or gene-cigarette smoking combination effects in relation to PCA risk.

\section{Competing interests}

The authors declare that they have no competing interests.

\section{Authors' contributions}

NAL, MLB, TTV and LRK designed and carried out genotyping assays and analyses; NAL, GNB, JHM and LRK performed statistical analyses; GNB, JHM, DWH and LRK served as mentors in designing and carrying out this project and editing the manuscript; All authors reviewed and edited the manuscript. All authors accepted the final manuscript.

\section{Acknowledgements}

The authors thank the men of African descent for their participation in the current study.

This work was supported by the National Cancer Institute R03 CA 28028 grant and the National Institute of Environmental Health Sciences T32 ESOII 564 and P30-ESOI4443 grants.

The authors appreciate access to the CGeMM DNA Core Facility at the UofL, directed by Dr. Ron Gregg, for high-throughput genotyping assays.

We thank Andrew Marsh at UofL's James Graham Brown Cancer Center for editing this manuscript.

\section{References}

I. American Cancer Society: Cancer Facts \& Figures 2009. 2009.

2. Mudashiru AS, Blessing E, Olapade-Olaopa EO: Update on prostate cancer in black men. J Men's Health \& Gender 2007, 4:456-463.

3. Powell II: Epidemiology and pathophysiology of prostate cancer in African-American men. J Urol 2007, $177: 444-449$.

4. Hsing AW, Chokkalingam AP: Prostate cancer epidemiology. Front Biosci 2006, I I:I388-14 I3.

5. Agalliu I, Langeberg WJ, Lampe JW, Salinas CA, Stanford JL: Glutathione S-transferase MI, TI, and PI polymorphisms and prostate cancer risk in middle-aged men. Prostate 2006, 66: | $46-156$.

6. Hayes JD, Strange RC: Glutathione S-transferase polymorphisms and their biological consequences. Pharmacology 2000 , 6I:154-166.

7. Mannervik B, Alin P, Guthenberg C, Jensson H, Tahir MK, Warholm $M$, et al.: Identification of three classes of cytosolic glutathione transferase common to several mammalian species: correlation between structural data and enzymatic properties. Proc Natl Acad Sci USA 1985, 82:7202-7206.

8. Ryberg D, Skaug V, Hewer A, Phillips DH, Harries LW, Wolf CR, et al.: Genotypes of glutathione transferase $M I$ and $P I$ and their significance for lung DNA adduct levels and cancer risk. Carcinogenesis 1997, 18:1285-1289. 
9. Ali-Osman F, Akande O, Antoun G, Mao JX, Buolamwini J: Molecular cloning, characterization, and expression in Escherichia coli of full-length cDNAs of three human glutathione Stransferase Pi gene variants. Evidence for differential catalytic activity of the encoded proteins. J Biol Chem 1997, 272: $10004-10012$.

10. Sundberg K, Johansson AS, Stenberg G, Widersten M, Seidel A, Mannervik $B$, et al.: Differences in the catalytic efficiencies of allelic variants of glutathione transferase PI-I towards carcinogenic diol epoxides of polycyclic aromatic hydrocarbons. Carcinogenesis 1998, 19:433-436.

II. Ntais C, Polycarpou A, loannidis JP: Association of GSTMI, GSTTI, and GSTPI gene polymorphisms with the risk of prostate cancer: a meta-analysis. Cancer Epidemiol Biomarkers Prev 2005, I4: |76-|8I.

12. Mo Z, Gao Y, Cao Y, Gao F, Jian L: An updating meta-analysis of the GSTMI, GSTTI, and GSTPI polymorphisms and prostate cancer: a HuGE review. Prostate 2009, 69:662-688.

13. Coughlin SS, Hall IJ: A review of genetic polymorphisms and prostate cancer risk. Ann Epidemiol 2002, I 2:182-196.

14. Lee E, Huang Y, Zhao B, Seow-Choen F, Balakrishnan A, Chan SH: Genetic polymorphism of conjugating enzymes and cancer risk: GSTMI, GSTTI, NATI and NAT2. J Toxicol Sci 1998, 23(Suppl 2): 140-142.

15. Sreelekha TT, Ramadas K, Pandey M, Thomas G, Nalinakumari KR, Pillai MR: Genetic polymorphism of CYPIAI, GSTMI and GSTTI genes in Indian oral cancer. Oral Oncol 200I, 37:593-598.

16. Katoh T, Inatomi H, Kim H, Yang M, Matsumoto T, Kawamoto T: Effects of glutathione S-transferase (GST) MI and GSTTI genotypes on urothelial cancer risk. Cancer Lett 1998, 132:147-152.

17. Salagovic J, Kalina I, Stubna J, Habalova V, Hrivnak M, Valansky L, et al: Genetic polymorphism of glutathione S-transferases MI and TI as a risk factor in lung and bladder cancers. Neoplasme 1998, 45:312-317.

18. Nakazato H, Suzuki K, Matsui $\mathrm{H}$, Koike $\mathrm{H}$, Okugi $\mathrm{H}$, Ohtake $\mathrm{N}$, et al:: Association of genetic polymorphisms of glutathione-Stransferase genes (GSTMI, GSTT I and GSTPI) with familial prostate cancer risk in a Japanese population. Anticancer Res 2003, 23:2897-2902.

19. Murata M, Watanabe M, Yamanaka M, Kubota $Y$, Ito $H$, Nagao $M$, et al: Genetic polymorphisms in cytochrome P450 (CYP) IAI, CYPIA2, CYP2EI, glutathione S-transferase (GST) MI and GSTTI and susceptibility to prostate cancer in the Japanese population. Cancer Lett 200I, 165:17I-I77.

20. Steinhoff C, Franke KH, Golka K, Thier R, Romer HC, Rotzel C, et al.: Glutathione transferase isozyme genotypes in patients with prostate and bladder carcinoma. Arch Toxicol 2000, 74:52I-526.

21. Komiya, , Tsukino H, Nakao H, Kuroda $Y$, Imai H, Katoh T: Human glutathione S-transferase AI, TI, MI, and PI polymorphisms and susceptibility to prostate cancer in the Japanese population. I Cancer Res Clin Oncol 2005, I 3 I:238-242.

22. Autrup JL, Thomassen LH, Olsen JH, Wolf H, Autrup H: Glutathione S-transferases as risk factors in prostate cancer. Eur J Cancer Prev 1999, 8:525-532.

23. Wadelius M, Autrup JL, Stubbins MJ, Andersson SO, Johansson JE, Wadelius $C$, et al.: Polymorphisms in NAT2, CYP2D6, CYP2CI9 and GSTPI and their association with prostate cancer. Pharmacogenetics 1999, 9:333-340.

24. Gsur A, Haidinger G, Hinteregger S, Bernhofer G, Schatzl G, Madersbacher S, et al:: Polymorphisms of glutathione-S-transferase genes (GSTPI, GSTMI and GSTTI) and prostate-cancer risk. Int I Cancer 200I, 95: I52-I55.

25. Beer TM, Evans AJ, Hough KM, Lowe BA, McWilliams JE, Henner WD: Polymorphisms of GSTPI and related genes and prostate cancer risk. Prostate Cancer Prostatic Dis 2002, 5:22-27.

26. Mallick S, Romana M, Blanchet P, Multigner L: GSTMI and GSTT I polymorphisms and the risk of prostate cancer in a Caribbean population of African descent. Urology 2007, 69:1165-1169.

27. Nock NL, Liu X, Cicek MS, Li L, Macarie F, Rybicki BA, et al.: Polymorphisms in polycyclic aromatic hydrocarbon metabolism and conjugation genes, interactions with smoking and prostate cancer risk. Cancer Epidemiol Biomarkers Prev 2006, | 5:756-76|.
28. Giri VN, Egleston B, Ruth K, Uzzo RG, Chen DY, Buyyounouski M, et al: Race, genetic West African ancestry, and prostate cancer prediction by prostate-specific antigen in prospectively screened high-risk men. Cancer Prev Res (Phila Pa) 2009, 2:244-250.

29. Brasch-Andersen C, Christiansen L, Tan Q, Haagerup A, Vestbo J, Kruse TA: Possible gene dosage effect of glutathione-S-transferases on atopic asthma: using real-time PCR for quantification of GSTMI and GSTTI gene copy numbers. Hum Mutat 2004, 24:208-2I4.

30. Packer BR, Yeager M, Burdett L, Welch R, Beerman M, Qi L, et al:: SNP500Cancer: a public resource for sequence validation, assay development, and frequency analysis for genetic variation in candidate genes. Nucleic Acids Res 2006, 34:D617-D621.

3I. Covault J, Abreu C, Kranzler H, Oncken C: Quantitative real-time PCR for gene dosage determinations in microdeletion genotypes. Biotechniques 2003, 35:594-6. 598

32. Tian C, Hinds DA, Shigeta R, Kittles R, Ballinger DG, Seldin MF: A genomewide single-nucleotide-polymorphism panel with high ancestry information for African American admixture mapping. Am J Hum Genet 2006, 79:640-649.

33. Hahn LW, Ritchie MD, Moore JH: Multifactor dimensionality reduction software for detecting gene-gene and gene-environment interactions. Bioinformatics 2003, 19:376-382.

34. Ritchie MD, Hahn LW, Moore JH: Power of multifactor dimensionality reduction for detecting gene-gene interactions in the presence of genotyping error, missing data, phenocopy, and genetic heterogeneity. Genet Epidemiol 2003, 24:150-157.

35. Hahn LW, Moore JH: Ideal discrimination of discrete clinical endpoints using multilocus genotypes. In Silico Biol 2004, 4:183-194.

36. Jakulin A, Bratko I: Analyzing attribute depedencies. Edited by: Lavrac N, Gamberger D, Blockeel H, Todorovski L. Cavtat, Croatia.: Springer-Verlag, PKDD; 2003:229-240.

37. Demosar J, Zupan B: Orange: From Experimental Machine Learning to Interactive Data Mining, White Paper 2004.

38. Andrew AS, Nelson HH, Kelsey KT, Moore JH, Meng AC, Casella DP, et al.: Concordance of multiple analytical approaches demonstrates a complex relationship between DNA repair gene SNPs, smoking, and bladder cancer susceptibility. Carcinogenesis 2006, 27: 1030-1037.

39. Jakulin A, Bratko I, Smrike D, Demsar J, Zupan B: Attribute interactions in medical data analysis. Protarus: Cyprus 2003:229-238.

40. National Center for Biotechnology Information (NCBI) website 2007 [http://www.ncbi.nlm.nih.gov]

4I. Nock NL, Liu X, Cicek MS, Li L, Macarie F, Rybicki BA, et al.: Polymorphisms in polycyclic aromatic hydrocarbon metabolism and conjugation genes, interactions with smoking and prostate cancer risk. Cancer Epidemiol Biomarkers Prev 2006, I5:756-76|.

42. Sikka SC: Role of oxidative stress response elements and antioxidants in prostate cancer pathobiology and chemoprevention--a mechanistic approach. Curr Med Chem 2003, 10:2679-2692.

43. Kelada SN, Kardia SL, Walker AH, Wein AJ, Malkowicz SB, Rebbeck TR: The glutathione S-transferase-mu and -theta genotypes in the etiology of prostate cancer: genotype-environment interactions with smoking. Cancer Epidemiol Biomarkers Prev 2000, 9:1329-1334.

44. Fleshner NE, Klotz LH: Diet, androgens, oxidative stress and prostate cancer susceptibility. Cancer Metastasis Rev 1998, I7:325-330

45. Abate-Shen C, Shen MM: Molecular genetics of prostate cancer. Genes Dev 2000, 14:24I0-2434.

46. Choi JY, Neuhouser ML, Barnett M, Hudson M, Kristal AR, Thornquist $M$, et al.: Polymorphisms in oxidative stress-related genes are not associated with prostate cancer risk in heavy smokers. Cancer Epidemiol Biomarkers Prev 2007, 16: I I I5- I I 20.

47. Waris G, Ahsan H: Reactive oxygen species: role in the development of cancer and various chronic conditions. J Carcinog 2006, 5:14.

48. Miyake $\mathrm{H}$, Hara I, Kamidono $\mathrm{S}$, Eto $\mathrm{H}$ : Oxidative DNA damage in patients with prostate cancer and its response to treatment. J Urol 2004, I 7 I:1533-1536.

49. Medeiros R, Vasconcelos A, Costa S, Pinto D, Ferreira P, Lobo F, et al: Metabolic susceptibility genes and prostate cancer risk in 
a southern European population: the role of glutathione Stransferases GSTMI, GSTM3, and GSTTI genetic polymorphisms. Prostate 2004, 58:4|4-420.

50. Kidd LR, Woodson K, Taylor PR, Albanes D, Virtamo J, Tangrea JA: Polymorphisms in glutathione-S-transferase genes (GSTMI, GST-TI and GST-PI) and susceptibility to prostate cancer among male smokers of the ATBC cancer prevention study. Eur J Cancer Prev 2003, I 2:3 I7-320.

5I. McCarty KM, Santella RM, Steck SE, Cleveland RJ, Ahn J, Ambrosone $\mathrm{CB}$, et al:: PAH-DNA adducts, cigarette smoking, GST polymorphisms, and breast cancer risk. Environ Health Perspect 2009, I I 7:552-558.

52. Mir O, Alexandre J, Tran A, Durand JP, Pons G, Treluyer JM, et al:: Relationship between GSTP I Ile(I05)Val polymorphism and docetaxel-induced peripheral neuropathy: clinical evidence of a role of oxidative stress in taxane toxicity. Ann Oncol 2009, 20:736-740.

53. Sreeja L, Syamala V, Hariharan S, Syamala VS, Raveendran PB, Sivanandan CD, et al: Glutathione S-transferase MI, TI and PI polymorphisms: susceptibility and outcome in lung cancer patients. J Exp Ther Oncol 2008, 7:73-85.

54. Rybicki BA, Neslund-Dudas C, Nock NL, Schultz LR, Eklund L, Rosbolt J, et al.: Prostate cancer risk from occupational exposure to polycyclic aromatic hydrocarbons interacting with the GSTP I Ile I05Val polymorphism. Cancer Detect Prev 2006, 30:412-422.

55. Loeb S, Catalona WJ: What is the prognostic impact of positive surgical margins in surgically treated prostate cancer? Nat Clin Pract Urol 2006, 3:418-419.

56. Shriver MD, Kittles RA: Genetic ancestry and the search for personalized genetic histories. Nat Rev Genet 2004, 5:6 I I-6I8.

57. Kittles RA, Chen W, Panguluri RK, Ahaghotu C, Jackson A, Adebamowo CA, et al.: CYP3A4-V and prostate cancer in African Americans: causal or confounding association because of population stratification? Hum Genet 2002, I I 0:553-560.

\section{Pre-publication history}

The pre-publication history for this paper can be accessed here:

http://www.biomedcentral.com/1471-2407/9/397/pre pub
Publish with Biomed Central and every scientist can read your work free of charge

"BioMed Central will be the most significant development for disseminating the results of biomedical research in our lifetime."

Sir Paul Nurse, Cancer Research UK

Your research papers will be:

- available free of charge to the entire biomedical community

- peer reviewed and published immediately upon acceptance

- cited in PubMed and archived on PubMed Central

- yours - you keep the copyright

Submit your manuscript here:

http://www.biomedcentral.com/info/publishing_adv.asp
BioMedcentral 